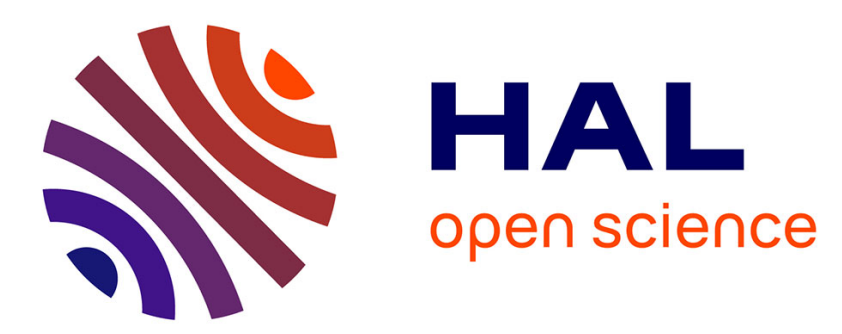

\title{
Quasiparticle lifetimes and tunneling times in an SS'IS"S tunnel junction detector
}

\author{
A. Golubov, E. Houwnan, V. Krasnov, J. Gijsbertsen, J. Flokstra, H. Rogalla, \\ J. Le Grand, P. de Korte
}

\section{- To cite this version:}

A. Golubov, E. Houwnan, V. Krasnov, J. Gijsbertsen, J. Flokstra, et al.. Quasiparticle lifetimes and tunneling times in an SS'IS"S tunnel junction detector. Journal de Physique IV Proceedings, 1994, 04 (C6), pp.C6-273-C6-278. 10.1051/jp4:1994644 . jpa-00253140

\section{HAL Id: jpa-00253140 https://hal.science/jpa-00253140}

Submitted on 1 Jan 1994

HAL is a multi-disciplinary open access archive for the deposit and dissemination of scientific research documents, whether they are published or not. The documents may come from teaching and research institutions in France or abroad, or from public or private research centers.
L'archive ouverte pluridisciplinaire HAL, est destinée au dépôt et à la diffusion de documents scientifiques de niveau recherche, publiés ou non, émanant des établissements d'enseignement et de recherche français ou étrangers, des laboratoires publics ou privés. 


\title{
Quasiparticle lifetimes and tunneling times in an SS'IS"S tunnel junction detector
}

\author{
A.A. Golubov(1), E.P. Houwnan, V.M. Krasnov(1), J.G. Gijsbertsen, J. Flokstra, H. Rogalla, \\ J.B. le Grand* and P.A.J. de Korte* \\ University of Twente, Department of Applied Physics, 7500 AE Enschede, The Netherlands \\ * Laboratory for Space Research, Sorbonnelaan 2, 3584 CA Utrecht, The Netherlands
}

\begin{abstract}
S}^{\prime}$ and $\mathbf{S}^{\prime \prime}$, near the tunnel barrier can be described as an SS'IS"S structure. Gap reduction takes place in S due to the proximity effect with the $S^{\prime}\left(S^{\prime \prime}\right)$ layer. Effective trapping, excitation and tunneling rates of the reduced gap region in the junction are calculated as a function of the operating temperature and junction bias voltage on the basis of a microscopic model of the proximity effect in the SS' sandwich. The calculations are done under the assumption that the thickness of the $S^{\prime}$ layer $d_{s^{\prime}}$ is small compared to its coherence length $\xi_{s^{\prime}}$ and dirty limit conditions are fulfilled for both the $S$ and $S^{\prime}$ metals. The limits of applicability of these approximations are discussed by calculating corrections to the space dependence of the order parameter in $S$ and $S^{\prime}$ due to a) finite thickness $d_{s^{\prime}}$ of the $S^{\prime}$ layer and b) for large mean free path $l_{s, s^{\prime}}$ in the $S$ and $S^{\prime}$ layers.
\end{abstract}

\section{INTRODUCTION}

An artificial trapping layer adjacent to the tunnel barrier in a superconducting tunnel junction based detector of high energy particles can be used advantageously to collect the excess quasipaticles (qp), created by the breaking of Cooper pairs after the absorption of the particle, from the absorber very fast and effectively $(1)$. Therefore the calculation of the time constants, which are relevant for the operation of such a device, is of high interest. We have considered a tunnel junction of SS'IS"S type as a model of the detector ( $\mathrm{S}$ is a bulk superconductor and $\mathrm{S}^{\prime}$ and $\mathrm{S}^{\prime \prime}$ are the trapping layers having smaller bulk energy gaps $\left.\mathrm{T}_{c s^{\prime}}, \mathrm{T}_{c s^{\prime \prime}} \leq \mathrm{T}_{c s}\right)$. The characteristic time constants in this problem are: effective quasiparticle scattering and recombination lifetimes for a proximity sandwich $\mathrm{SS}^{\prime}$ and effective tunneling times for electrons and holes tunneling out of the trap in the SS'-sandwich to the other electrode of the junction. These calculated time constants can be applied straightforwardly in the Rothwarf-Taylor equations for the number densities of non-equilibrium quasiparticles and phonons to describe non-equilibrium processes in the tunnel junction detector (2).

In this paper we will give some results of a model from which the effective time constants are calculated as a function of temperature, voltage over the junction, and strength of the proximity effect for the case of a dirty metal sandwich $\left(l_{s, s^{\prime}}<<\xi_{s, s^{\prime}}\right)$ with thin $S^{\prime}$ layer $\left(d_{s^{\prime}}<<\xi_{s^{\prime}}\right)$, where $l_{s, s^{\prime}},\left(\xi_{s, s^{\prime}}\right)$ are the mean free path $(\mathrm{mfp})$ and coherence length in $S\left(S^{\prime}\right)$, respectively. The dependence of the results on the $S^{\prime}$ layer thickness $d_{s^{\prime}}$ and the applicability of the thin $S^{\prime}$-layer approximation will be discussed. Effects due to large $\mathrm{mfp}$ in the $S$ and $\mathrm{S}^{\prime}$ layers (the clean limit) will be considered.

(1) On leave from the Institute of Solid State Physics, 142432 Chernogolovka, Moscow district, Russia 


\section{MODEL OF THE PROXIMITY EFFECT}

To describe the proximity effect in an $S^{\prime} S^{\prime}$ sandwich of dirty superconductors $S$ and $S^{\prime}$ the McMillan model(3) was used before extensively. However it is applicable only for the case of a large potential barrier at the SS' interface and thin $S$ and $S^{\prime}$ layers $\left(d_{s, s^{\prime}}<<\xi_{s, s^{\prime}}\right)$. For the more general case of arbitrary transparency of the SS' interface a microscopic model of the proximity effect was developed recently $(4,5)$ for the case of a bulk $S$ and a thin $S^{\prime}$ layer. Short electron mean free paths were assumed in both $S$ and $S^{\prime}$. In this case the proximity effect between $S$ and $S^{\prime}$ is described with three parameters, $\gamma_{B}=(2 / 3)\left(l_{s^{\prime}} / \xi_{s^{\prime}}\right)\left(d_{s^{\prime}} / \xi_{s^{\prime}}\right)<(1-D) / D>, \gamma_{m}=\left(\rho_{s} \xi_{s} / \rho_{s^{\prime}} \xi_{s^{\prime}}\right)\left(d_{s^{\prime}} / \xi_{s^{\prime}}\right)$ and the critical temperature ratio $T_{c s^{\prime}} / T_{c s}$. Here $\rho$ is the normal state resistivity, $\xi$ the coherence length, and $D$ the transmission coefficient of the SS' boundary. In the practically important limit of good electrical contact between $S$ and $S^{\prime}$, i.e. opposite to that of McMillan, the transmission coefficient $D \approx 1$, thus $\gamma_{B} \approx 0$. Most of the calculations in this paper are done for a $\mathrm{Nb} / \mathrm{Al}$ sandwich with $T_{c s^{\prime}} / T_{c s}=0.14$, but a variation of the $T_{c s^{\prime}} / T_{c s}$ ratio for other materials can be taken into account by renormalization of $\gamma_{m}(4,5)$.

The time constants of a spatially homogeneous superconductor were calculated by Kaplan et al. (6). The generalization to the inhomogenous case, and in particular for an SS' sandwich, was done in refs. $(7,8)$. As was shown, the time constants can be expressed through the normalized qp and pair densities of states $\overline{N(\varepsilon, x)}$ and $\overline{P(\varepsilon, x)}$ in the $\mathrm{S}$ and $\mathrm{S}^{\prime}$ layers. Therefore one should first solve the proximity effect problem in order to calculate $\overline{N(\varepsilon, x)}$ and $\overline{P(\varepsilon, x)}$. The solutions for $\overline{N(\varepsilon)}$ in the $\mathrm{S}^{\prime}$ layer of the SS' sandwich are shown in fig. 1 for different values of the $\gamma_{m}$ parameter. It is seen that the energy gap in $\mathrm{S}^{\prime}$ is largely reduced with an increase of $\gamma_{m}$, but remains finite. For large $\gamma_{m}$ values the gap $\Delta_{g}$ becomes equal to the bulk value of the gap of the $S^{\prime}$ material, $\Delta_{o}{ }^{\prime}(0)$. The $\Delta_{g}\left(\gamma_{m}\right)$ decrease takes place also in the $\mathrm{S}$ layer at distances of the order of $\xi_{s}$ from the SS' boundary. This gap suppression is the main physical reason for the increase of the qp trapping rate in the SS' system, as well as of the increase of the

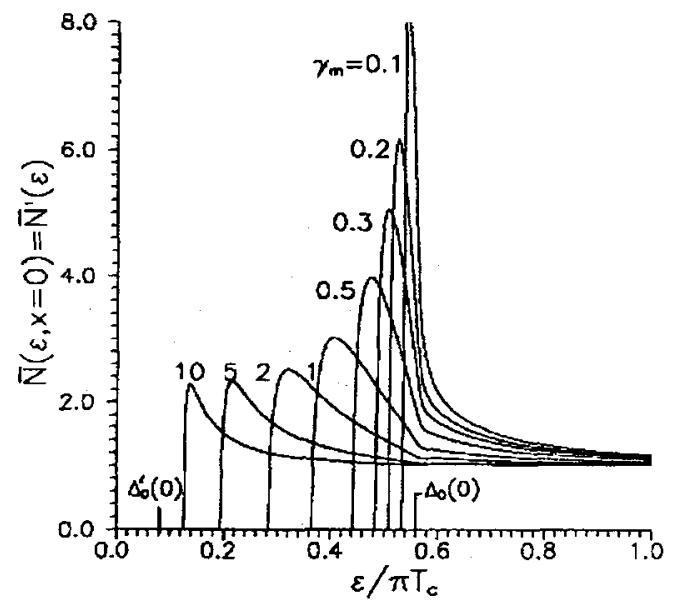

Fig. 1. The densities of states at the SS' interface for a sandwich with $\mathrm{T}_{\mathrm{cs}^{\prime} / \mathrm{T}_{\mathrm{c}}=0.14}$ (corresponding to a Nb/Al sandwich)

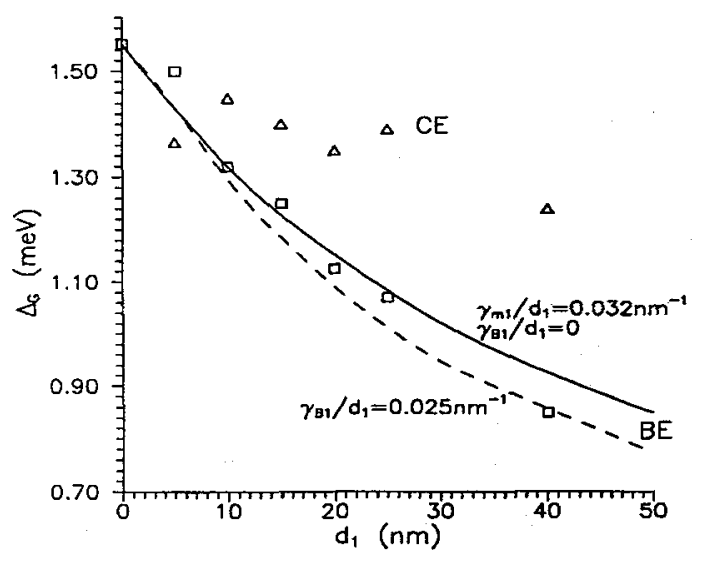

Fig.2. Measured energy gap ( $\square$ ) as function of the Al-layer thickness $d_{1}$ at the base electrode $(\mathrm{BE})$ and at the counterelectrode (CE) $(\Delta)$ with $\mathrm{d}_{2} \approx 3 \mathrm{~nm}$ 
tunneling rate, as will be discussed below. As an illustration we show the application of the thin-layer model for describing the gap reduction at low temperatures in the electrodes of a $\mathrm{Nb} / \mathrm{Al}_{1}, \mathrm{AlOx}, \mathrm{Al}_{2} / \mathrm{Nb}$ tunnel junction, in which the thickness $d_{1}$ of the $\mathrm{Al}_{1}$-layer was varied whereas that of the $\mathrm{Al}_{2}$-layer was approximately kept constant. The solid curve in fig. 2 gives a fit to the model with $\gamma_{m 1} / d_{1}=0.032 \mathrm{~nm}^{-1}$ and $\gamma_{B 1}=0$, the dashed one with the same $\gamma_{m 1} / d_{1}$ but with $\gamma_{B 1} / d_{1}=0.025 \mathrm{~nm}^{-1}$. Taking into account nonzero $\gamma_{B 1}$ gives a good quantitative agreement also for the thickest layers of $40 \mathrm{~nm}$.

\section{QUASIPARTICLE TRAPPING AND TUNNELING TIMES}

The trapping rate for the qp due to energy relaxation in the reduced gap region in $\mathbf{S}$ and $\mathrm{S}^{\prime}$ is defined as the rate by which the number of qp with energy $\varepsilon>\Delta_{0}, N_{>}^{t o t}$, is changed due to phonon emission:

$$
\frac{1}{\tau_{t r}}=\frac{1}{N_{>}^{t o t}}\left|\frac{d N_{>}^{t o t}}{d t}\right|=\frac{1.82}{d_{s}}\left[\frac{L_{e f f}\left(T, \gamma_{m}\right)}{\tau_{0}}+\beta \frac{L_{e f f}^{\prime}\left(T, \gamma_{m}\right)}{\tau_{0}^{\prime}}\right]
$$

It can be shown that the reduced gap region in $S$ respectively $S^{\prime}$ can be modeled as a trap with zero gap and width $L_{e f f}\left(L_{e f f}\right)$ and constant relaxation rate $\tau_{\varepsilon}^{-1}\left(\Delta_{g}=0\right)=1.82 / \tau_{0}$, where $\tau_{0}$ is a material dependent parameter of $S\left(S^{\prime}\right)$ as defined by Kaplan et al. (6). The effective length $L_{e f f}(T)$ is obtained by averaging the spatially and energy dependent relaxation rate over the qp density at a certain position and over the sandwich thickness. The factor $\beta$ is equal to $\left(v_{F} / v_{F}^{\prime}\right)\left(B^{\prime} / B\right)$, with $v_{F}\left(v_{F}^{\prime}\right)$ the Fermi- velocity of $\mathrm{S}\left(\mathrm{S}^{\prime}\right) . \quad \mathrm{B}^{\prime} / \mathrm{B}$ is nearly equal 1 for $\gamma_{m} \ll<1$ and equal $\left(2 k_{B} T / \pi \Delta_{0}\right)^{1 / 2}$ for $\gamma_{m}>1$ and low T. For $\mathrm{Nb} / \mathrm{Al}$ sandwich it was shown that the trapping is dominated by the $\mathrm{Nb}$ layer $(7)$ due to the much smaller timeconstant $\tau_{0}$ of $\mathrm{Nb}$ than that of $\mathrm{Al}$.

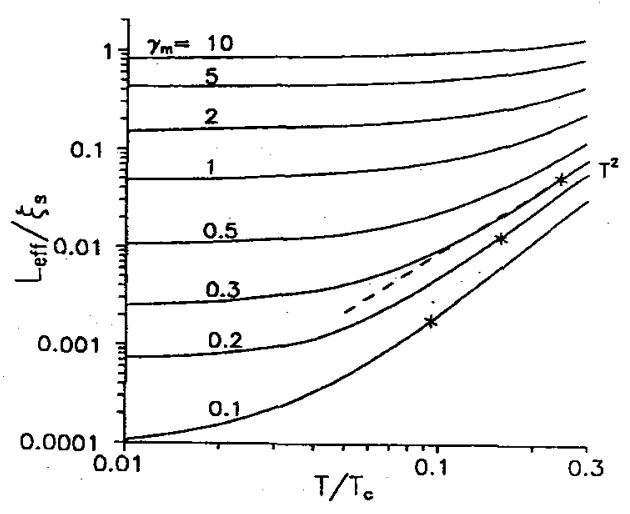

Fig.3. Temperature dependence of the effective trapping length of an $\mathrm{SS}^{\prime}$ sandwich $\left(\mathrm{T}_{\mathrm{cs}}{ }^{\prime} / \mathrm{T}_{\mathrm{cs}}=0.14\right)$ for different $\gamma_{m}$ values

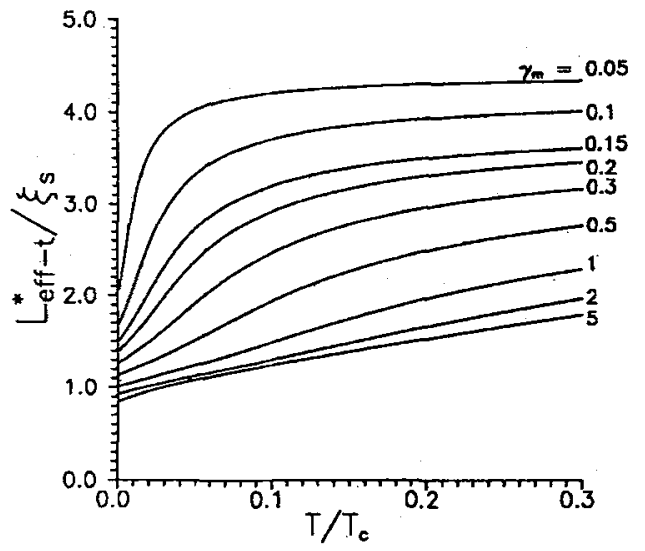

Fig.4. Temperature dependence of the effective tunneling length of the trap in the $S$ layer in an $\mathrm{SS}^{\prime}$ sandwich $\left(\mathrm{T}_{\mathrm{cs}} / \mathrm{T}_{\mathrm{cs}}=0.14\right)$ for different $\gamma_{m}$ values 
In fig.3 $L_{e f f}(T) / \xi_{s}$ is given as a function of temperature for different values of $\gamma_{m}$. It is seen that $L_{e f f}$ is practically constant at low temperatures and its value only depends on $\Delta_{g}$ determined by $\gamma_{m}$. $L_{e f f}$ increases with increasing $\gamma_{m}$ due to the gap suppression (see fig.1) saturating at a constant value for $\gamma_{m} \rightarrow \infty$ because $\Delta_{g}$ reaches $\Delta_{s^{\prime}}^{B C S} . L_{e f f}$ also increases with increasing temperature due to the thermal activation of the relaxation rate. $L_{\text {eff }}$ is the material independent parameter and therefore fig. 3 can be used to estimate a trapping rate for any combination of materials with various $T_{c s^{\prime}} / T_{c s}$ ratios as explained in more detail in $(4,7,8)$.

The tunneling time of the junction gives the rate with which a quasiparticle tunnels from one electrode to the other. The advantage of a trapping layer is that it reduces the tunneling time largely due to the smaller thickness of the trap compared to that of the bulk. The tunneling rate has to be considered separately for electron and hole-like qps. In the low temperature regime the effective tunneling time for electrons becomes

$$
\tau_{\text {tun }}^{(e)}(1 \rightarrow 2)=e^{2} R_{N} A^{\prime} \frac{N_{1}(0) L_{e f f}^{*} t}{\left(\gamma_{m}, T\right)+N_{1}^{\prime}(0) d_{1}}
$$

where $\mathrm{A}^{\prime}$ is the area of $\mathrm{S}^{\prime}$ and $R_{N}$ is the junction resistance. The effective tunneling length $L_{e f f_{-} t}^{*}$ depends only on temperature and $\gamma_{m}$, whereas the voltage dependence of the tunneling rate, $\tau_{\text {tun }}^{-1}$, is determined by the density of final states $\overline{N_{2}}$ to which the qps tunnel. In the spatially homogeneous case $L_{\text {eff_t }}^{*}=d_{s}$, then for large voltages $\overline{N_{2}} \cong 1$ and the well-known Ginsberg equation $(9) \tau_{t u n}^{(e)}(1 \rightarrow 2)=e^{2} R_{N} N_{1}(0) V_{s 1}$ is obtained (where $V_{s 1}=A^{\prime} d_{s 1}$ is the volume of electrode 1).

In fig.4 $L_{e f f_{-} t}^{*}$ is shown as a function of temperature for different $\gamma_{m}$. For small $\gamma_{m}$ the shallow trap is quickly filled with qps when temperature increases, and as a result the average length from which qps tunnel, $L_{\text {eff } t}^{*}$, also increases untill the trap is filled and $L_{\text {eff } t}^{*}$ saturates. The qps in the deep trap are confined to the narrow bottom of the trap, which becomes filled only slowly.

It can be shown that the tunneling time for holes is related to that of electron-like qps by the relation $\tau_{t u n}^{(h)}(1 \rightarrow 2)(V, T)=\tau_{\text {tun }}^{(e)}(1 \rightarrow 2)(-V, T)$, and the total current of positive charge tunneling from electrode 1 to electrode 2 is thus $I_{t o t}(1 \rightarrow 2)=I^{(h)}-I^{(e)}$. At low temperatures and voltages $I^{(h)}(1 \rightarrow 2)$ vanishes and the total current is mainly due to electrons, and according to eq.(1) its voltage dependence reflects therefore the density of states of the electrode $2, \bar{N}_{2}\left(\Delta_{1 g}+e V\right)$. In fig.5 the electron current $I(1 \rightarrow 2)$ is shown (a) calculated exactly (solid lines) and (b) according to the approximate relation (2) (dotted lines). It is seen that the approximate dependence (2) gives a reasonably good approximation for the tunnel current and can therefore be used to estimate the tunneling rate at finite voltages. For quantitative estimates one shall use eq.(2) substituting the function $\bar{N}_{2}\left(\Delta_{1 g}+e V\right)$ by the exact dependence as shown in fig. 5 , normalized to 1 for large eV. The tunneling rates from electrode 2 to 1 can be derived in the same way. In fig.5 the tunneling currents $I^{(e)}(2 \rightarrow 1) \propto 1 / \tau_{\text {tun }}^{(e)}(2 \rightarrow 1)$ and 
$I^{(h)}(2 \rightarrow 1) \propto 1 / \tau_{\text {tun }}^{(h)}(2 \rightarrow 1)$, as well as the sum current $I_{t o t}(2 \rightarrow 1)=I^{(h)}-I^{(e)}$ of positive charge from electrode 2 to 1 are shown. The approximation due to $\overline{N_{1}}\left(\Delta_{2 g}-e V\right)$ is shown by the dashed line. These curves can also be used to determine the voltage dependence of tunneling rate from 2 to 1 .
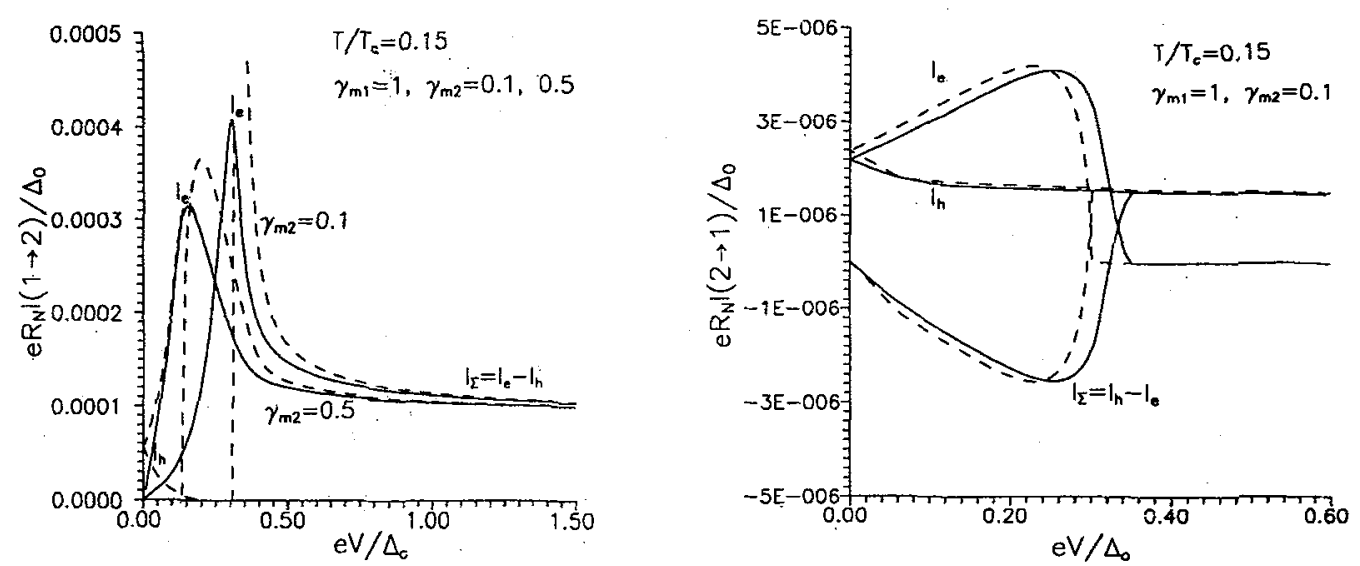

Fig.5. Tunneling rates for electrons and holes from the electrode 1 to 2 (left) and from 2 to 1 (right). The dashed lines give the approximate dependence through $\overline{N_{2}}$.

\section{GENERALIZATION OF THE MODEL}

The solutions of the proximity effect model discussed above were obtained in the limit of a thin proximity layers, $d / \xi_{s^{\prime}}<<1$ and short electron mean free paths in $S$ and $S^{\prime}$-layer. Here we discuss qualitatively the deviations from this model when (a) the proximity layer $S^{\prime}$ is thick, (b) both $S$ and $S^{\prime}$ are in the clean limit (large electron mfp).

To derive the criterion of the applicability for case (a) we have solved the equations of the proximity effect model numerically for the $\mathrm{SN}$-case $\left(\mathrm{T}_{c s^{\prime}}=0\right)$ for arbitrary $\mathrm{N}$-layer thickness. In fig. 6 we show the results for the superconducting order parameter at the SN boundary, $\Delta_{s}(0)$, calculated in the thin-layer approximation with $\gamma_{m}=\gamma d_{N} / \xi_{N}$ and $\gamma_{B}=\gamma_{B N} d_{N} / \xi_{N} \quad$ (dotted lines) and using the exact solution (solid lines) for the same values of $\gamma$ and $\gamma_{B N}$. For thicknesses $d_{N} / \xi_{N} \leq 0.5$ the corrections are less than $20 \%$ for the $\gamma_{B N}=0$ case, and even smaller for finite $\gamma_{B N}$ values, whereas for larger $\mathrm{N}$-layer thicknesses it is necessary to use the exact solutions of the model in order to obtain quantitative results. Therefore we consider $d_{s^{\prime}} / \xi_{s^{\prime}} \leq 0.5$ to be a good criterion of applicability for the calculations in section 3 .

If both $S$ and $S^{\prime}$ metals are in the clean limit, then the situation depends on the scattering properties of the SS' boundary. Two qualitatively different limits are to be considered : diffusive scattering and mirror-reflecting scattering. In the first case, which is realized in most of presently made junctions, the dirty limit theory is still valid, at least for thin layers, due to boundary scattering. In the second case important modifications take place. In fig. 7 the solutions for the order parameter in $\mathrm{S}$ due to the proximity effect in a SN sandwich are shown for various values of the parameter $\gamma_{c l}=\left(d_{N} / \xi_{s}\right) N_{s}(0) / N_{N}(0)$ along with the solutions of the dirty limit model for various $\gamma_{m}$, where both parameters are related as : $\gamma_{c l}=\gamma_{m}$ for $N_{s}(0) / N_{N}(0)>1$, and $\gamma_{c l}=\gamma_{m}\left(N_{s}(0) / N_{N}(0)\right)^{2}$ for $N_{s}(0) / N_{N}(0)<1$. It is seen that for equal 


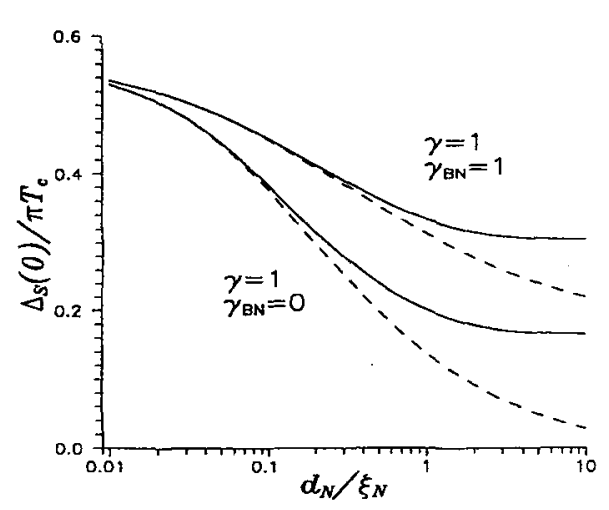

Fig.6. Order parameter at the interface of an SN sandwich in the $d_{N} / \xi_{N} \ll<$ approximation (dashed) and according to the exact solutions (solid).

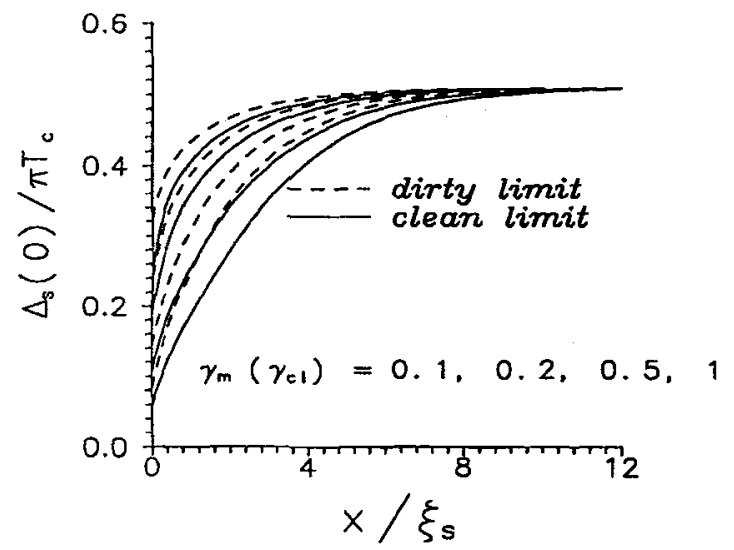

Fig.7. Comparison of the order parameter behaviour near the SN boundary for the dirty and clean limits.

values of pair-breaking parameters $\gamma_{m}$ and $\gamma_{c l}$ the order parameter in $\mathrm{S}$ is suppressed stronger in the clean case due to the larger coherence length in comparison with the dirty case. However, for the case $N_{s}(0) / N_{N}(0)<1$ this effect is partly compensated by the difference between $\gamma_{m}$ and $\gamma_{c l}$, i.e. with decreasing $N_{s}(0) / N_{N}(0)$ the S-layer becomes less effective as a trap. Therefore, materials with smaller density of states at the Fermi level, $N_{N}(0)$, are preferable for creating a trap. More detailed calculations of the dependence of the time constants on the ratio of the densities of states in the clean limit will be given elsewhere.

\section{ACKNOWLEDGMENTS}

This work in the program of the Foundation for Fundamental Research on Matter (FOM) is funded by the Netherlands Technology Foundation (STW).

\section{REFERENCES}

[1] Booth N.E., Appl.Phys.Lett. 50 (1987) 93.

[2] Rothwarf A. and Taylor B.N., Phys.Rev.Lett. 19 (1967) 27.

[3] McMillan W.L., Phys.Rev.B 175 (1968) 537.

[4] Golubov A.A. et al., JETP 76 (1993) 915.

[5] Golubov A.A. et al., submitted to Phys.Rev.B.

[6] Kaplan S.B. et al., Phys.Rev.B 14 (1976) 4854.

[7] Golubov A.A. et al. Phys.Rev.B, 49 May (1994);

Golubov A.A. and Houwman E.P., Physica C 205 (1993) 147.

[8] Houwman E.P. et al., J. Low Temp.Phys. 93 (1993) 67.

[9] Ginsberg D.M., Phys.Rev.Lett. 8, 204 (1962). 